Onkologe 2020 $26: 1056-1064$ https://doi.org/10.1007/s00761-020-00854-y Online publiziert: 21. Oktober 2020 (c) Der/die Autor(en) 2020

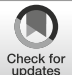

\author{
Marco Zschoche ${ }^{1} \cdot$ Steffen Emmert ${ }^{2}$. Nikolas von Bubnoff ${ }^{3}$ Mahdy Ranjbar ${ }^{1}$. \\ Salvatore Grisanti' ${ }^{1}$ Ludwig M. Heindl $^{4,5} \cdot$ Falko Fend $^{6} \cdot$ Irenäus A. Adamietz ${ }^{7}$. \\ Vinodh Kakkassery ${ }^{1}$ \\ ' Klinik für Augenheilkunde, Universitätsklinikum Schleswig-Holstein, Campus Lübeck, Lübeck, \\ Deutschland \\ ${ }^{2}$ Klinik und Poliklinik für Dermatologie und Venerologie, Universitätsmedizin Rostock, Rostock, \\ Deutschland \\ ${ }^{3}$ Klinik für Hämatologie und Onkologie, Universitätsklinikum Schleswig-Holstein, Campus Lübeck, Lübeck, \\ Deutschland \\ ${ }^{4}$ Zentrum für Augenheilkunde, Universität zu Köln, Medizinische Fakultät, Universitätsklinikum Köln, Köln, \\ Deutschland \\ ${ }^{5}$ Centrum für Integrierte Onkologie (CIO) Aachen - Köln - Bonn - Düsseldorf, Köln, Deutschland \\ ${ }^{6}$ Institut für Pathologie und Neuropathologie, Comprehensive Cancer Center Tübingen-Stuttgart, \\ Universitätsklinikum Tübingen, Eberhard-Karls-Universität Tübingen, Tübingen, Deutschland \\ ${ }^{7}$ Universitätsklinik für Radioonkologie, Ruhr-Universität Bochum, Bochum, Deutschland
}

\title{
Augenbefall und Systemerkrankung - periokuläre und intraokuläre Lymphome
}

zeichnet ein Rezidiv oder die Manifestation eines Lymphoms durch hämatogene/ lymphogene Aussaat, ausgehend von einer systemischen Lymphomerkrankung [19]. Eine weitere Besonderheit leitet sich aus der paarigen Anlage der Augen ab, sodass entweder unilateral oder bilateral die Augen betroffen sein können.

Vitreoretinale Lymphome werden den ZNS-Lymphomen zugerechnet, sind überwiegend diffus-großzellige B-ZellLymphome und haben eine schlechte Prognose, während primäre Lymphome der Choroidea, periokuläre Lymphome (auch Lymphome der okulären Adnexe) überwiegend indolenten extranodalen Marginalzonenlymphomen mit günstiger Prognose entsprechen (• Abb. 1). - Tab. 1 und 2 zeigen die Charakteristika der periokulären und intraokulären Lymphome (- Tab. 1 und 2).

Große Bedeutung kommt dieser Erkrankung aufgrund einer konstant steigenden Inzidenz über die vergangenen Jahrzehnte $\mathrm{zu}$, welche bei bis $\mathrm{zu}$ 6,5\% jährlich lag. Die steigende Lebenserwartung, verbesserte diagnostische Methoden sowie die ansteigende Prävalenz von Immunerkrankungen werden als mögliche Ursachen dafür diskutiert [35]. In den meisten Fällen erkranken Menschen nach dem 60. Lebensjahr. In Deutschland geht die Prognose für 2020 von 22.900 Lymphomfällen aus [41]. Legt man die o.g. Daten zugrunde, würde der Anteil okulärer Lymphome in Deutschland jährlich bei ca. 500-700 Fällen liegen, wobei etwa 450-630 auf periokuläre Lymphome entfielen und ca. 50-70 auf intraokuläre Lymphome.

\section{Periokuläre Lymphome}

Die periokulären Lymphome beinhalten den Befall von Orbita, Konjunktiva, Lid sowie des Tränenapparats. Der orbitale und konjunktivale Befall zusammen machen ca. 70\% der Fälle aus [49].

In der Mehrheit der Fälle treten diese Lymphome primär auf. Sekundärer Befall kommt in bis zu 35\% der Fälle vor und zeigt sich häufig mit einer schlechteren Prognose [33]. Ein bilateraler Befall tritt in ca. $20 \%$ der Fälle auf und geht gehäuft mit einer systemischen Lymphomerkrankung einher [33, 47, 49]. Bei primärer Manifestation tritt klassischerweise das niedrigmaligne extranodale Marginalzonenlymphom (MALT-Lymphom oder EMZL) am häufigsten auf, wäh- 

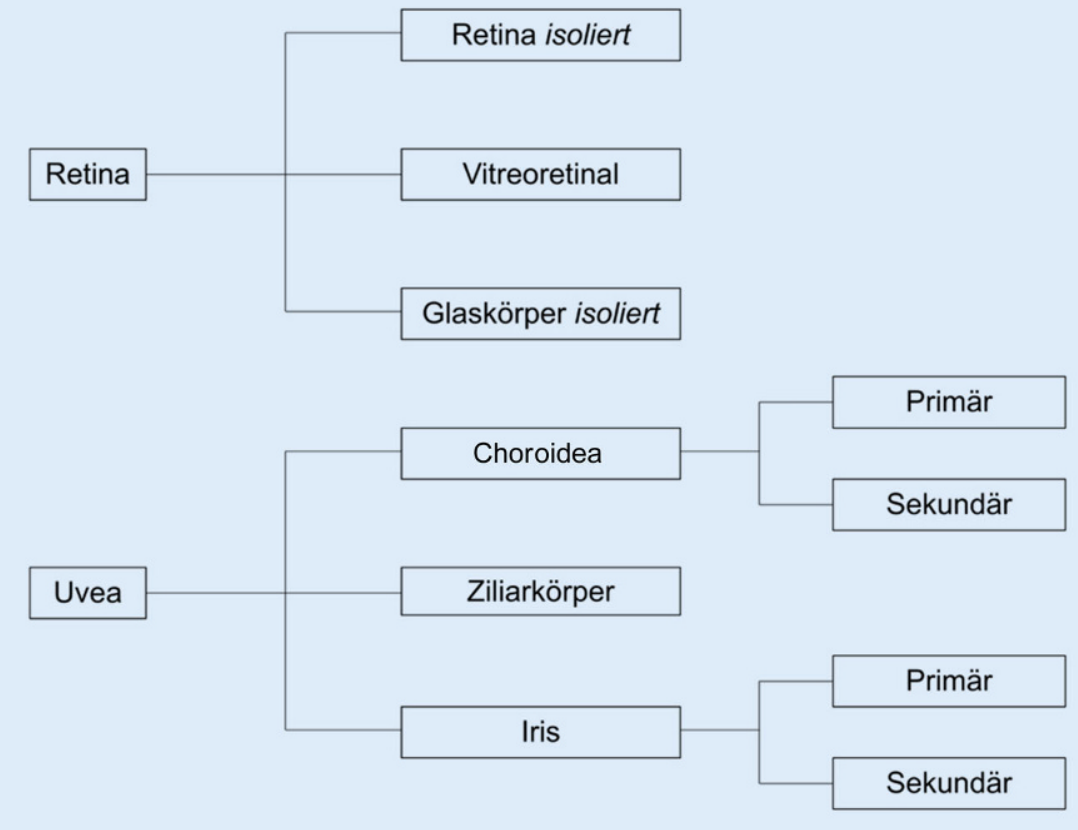

Abb. 1 \ Einteilung intraokulärer Lymphome nach anatomischer Lokalisation. Das intraokuläre Lymphom wird anhand des anatomischen Befalls eingeteilt. Man unterscheidet grob die Lymphome, welche die Uvea befallen, von denen, die die Retina betreffen. An der Retina unterscheidet man den isolierten Befall der Retina oder des Glaskörpers vom häufiger auftretenden kombinierten Befall der Retina und des Glaskörpers als vitreoretinales Lymphom. Die uvealen Lymphome werden weiter in choroidale Lymphome, Ziliarkörperlymphome und Irislymphome unterteilt, wobei man vor allem bei den choroidalen und Irislymphomen weiter zwischen primärem und sekundärem Befall unterscheidet. Choroidale Lymphome treten gehäuft als primärer Befall auf, wohingegen Irislymphome typischerweise im Rahmen eines sekundären Befalls auftreten. Bei einem kombinierten Befall der verschiedenen Strukturen wird das Lymphom nach dem größeren Befund an der anatomischen Struktur benannt. (Angepasst übernommen von [19])

rend bei sekundärem Befall diffus-großzelliges B-Zell-Lymphom (DLBCL), follikuläres Lymphom (FL), Mantelzelllymphom (MCL) und die chronische lymphatische Leukämie (CLL) die häufigsten Subtypen darstellen [49]. Die Assoziation der adnexalen MALT-Lymphome mit dem Nachweis von Chlamydia psittaci im Tumorgewebe wird kontrovers diskutiert; möglicherweise spielen geographische Faktoren eine Rolle bei den unterschiedlichen Studienergebnissen [23]. Auffällig ist bei Lymphomen des Augenlids der im Vergleich zu den anderen Lokalisationen höhere Anteil an peripheren T-Zell-Lymphomen, was auf dem Befall der Lider durch kutane T-ZellLymphome beruht (• Abb. 2; [43]).

Die klinische Symptomatik periokulärer Lymphome ist heterogen. Die Diagnose wird teils erst nach Monaten oder Jahren gestellt. Beim Befall der Orbita zeigt sich häufig eine Protrusio bul-
Neben dem Abstrich ist es notwendig, bekannte Koinzidenzen wie Hepatitis C oder HIV sowie Autoimmunerkrankungen wie das Sjögren-Syndrom abzuklären [36]. Bildgebend stellt die MRT den Goldstandard dar. Die endgültige Diagnosestellung kann jedoch nur durch die histopathologische Untersuchung einer Biopsie erfolgen. Nach Diagnosesicherung sollte ein klinisches Staging erfolgen. Dieses ist wichtig, um festzustellen, ob ein primärer lokalisierter Befall vorliegt oder sich die Lymphomerkrankung schon ausgebreitet hat. Bildgebend sollte dazu die MRT oder PET-CT eingesetzt werden. Auch eine Knochenmarkbiopsie zum Ausschluss einer Knochenmarkinfiltration sollte erfolgen [22].

Nach der Diagnostik ist die Vorstellung im Tumorboard mit den erforderlichen Fachdisziplinen zur Therapiefestlegung unerlässlich. Die Therapie richtet sich nach dem Ausmaß der Erkrankung und systemischen Beteiligung. Die Behandlungsoptionen reichen von radiotherapeutischen Verfahren über die systemische sowie lokale Anwendung von Chemotherapeutika bzw. Immuntherapeutika bis hin zu einer Watchand-wait-Strategie (vor allem bei älteren Menschen) und Therapieversuchen durch den Einsatz von Antibiotika. Auch kombinierte Therapieverfahren kommen zum Einsatz. Die alleinige chirurgische Resektion ist durch die besseren therapeutischen Erfolge der anderen Therapiemethoden in den Hintergrund geraten. Als effektive und zugleich nebenwirkungsarme Therapie hat sich die Strahlentherapie etabliert. Sie wird als First-line-Therapie vor allem bei lokalisiertem niedrigmalignem $\mathrm{Be}$ fall angewendet $[22,38]$. - Abb. 3 stellt einen konjunktivalen Lymphombefund vor und nach Bestrahlung dar (• Abb. 3). Ein wesentlicher Vorteil der Therapie ist aufgrund der langen Etablierung die beste Datenlage am Auge im Vergleich zu den anderen Therapien [14].

Hochmaligne Lymphome sowie auch der systemische Befall niedrigmaligner Lymphome werden meist durch Chemotherapie oder Immunchemotherapie behandelt, u.a. auch bei Rezidiven nach vorheriger anderer Therapie [38]. Vorteile sind hierbei vor allem die Vermei- 
Onkologe 2020 $26: 1056-1064$ https://doi.org/10.1007/s00761-020-00854-y

(c) Der/die Autor(en) 2020

M. Zschoche · S. Emmert · N. von Bubnoff · M. Ranjbar · S. Grisanti · L. M. Heindl · F. Fend · I. A. Adamietz · V. Kakkassery

Augenbefall und Systemerkrankung - periokuläre und intraokuläre Lymphome

\section{Zusammenfassung}

Hintergrund. Das okuläre Lymphom wird anhand seiner anatomischen Lokalisation in die intraokulären und periokulären Lymphome eingeteilt. Intraokulär kann die Uvea mit ihren Strukturen betroffen sein oder die Retina in Verbindung mit dem Glaskörper. Die periokulären Lymphome treten in Orbita, Bindehaut, Tränenapparat oder Lid auf. Von großer Bedeutung ist die Unterscheidung zwischen primären Lymphomen der Region oder systemischem Befall. Über die letzten Jahrzehnte konnte in den westlichen Ländern eine konstant steigende Inzidenz okulärer Lymphome nachgewiesen werden.

Ziel. Dieser Beitrag soll einen Überblick über die vielfältigen Manifestationen, Diagnostik,
Therapie sowie Prognose und Nachsorge geben.

Material und Methoden. Der Beitrag basiert auf einer selektiven Literaturrecherche über die MEDLINE-Datenbank zum Thema okuläre Lymphome sowie den persönlichen Erfahrungen der Autoren.

Ergebnisse. Je nach Lokalisation können die Symptome sehr unterschiedlich sein. Die Diagnose erfolgt über eine Probebiopsie und anschließende zytologische/histologische und ggf. molekularpathologische Untersuchung. Strahlentherapeutische sowie systemische Verfahren stellen die am häufigsten angewendeten Therapieverfahren dar. Die Prognose hängt sehr stark von der Lokalisation, dem
Subtyp des Lymphoms sowie dem Ausmaß des Tumorbefalls ab.

Diskussion. Das okuläre Lymphom berührt in Diagnostik, Therapie und Nachsorge die Schnittstellen zwischen Ophthalmologie, (Hämato-)Onkologie, Strahlentherapie, Neurologie, Neurochirurgie, Mund-KieferGesichts-Chirurgie, Hals-Nasen-OhrenHeilkunde, Dermatologie, Radiologie, Pathologie und Psychoonkologie. Dabei spielt der Augenarzt als Eingangsarzt bei dieser Systemerkrankung eine wesentliche Rolle.

Schlüsselwörter

Lymphome der okulären Adnexe - Choroidale Lymphome · Irislymphom · Ziliarkörperlymphom · Vitreoretinale Lymphome

\section{Between the poles of localized and systemic disease-Periocular and intraocular lymphoma}

\section{Abstract}

Background. Ocular lymphoma is classified as intraocular lymphoma and periocular lymphoma based on the anatomic location. Intraocularly, the uvea with its structures can be affected or the retina in combination with the vitreous body. Periocular lymphoma can occur in the orbit, the conjunctiva, the lacrimal apparatus, or the eye lid. The differentiation between primary lymphomas of the ocular region and systemic disease is of exceptional importance. Over the last few decades, the incidence has risen constantly in Western countries.

Aim. An overview of the heterogeneous manifestations, diagnostics, treatment strategies, prognosis, and aftercare of patients with ocular lymphoma is given.

Materials and methods. This review is based on a selective literature search of the MEDLINE database and on the authors' personal experience with this disease.

Results. Depending on the location of the tumor, the signs and symptoms can be very diverse. Diagnosis is based on biopsy and subsequent cytologic/histopathologic and when appropriate molecular pathologic examinations of the tissue. Radiation therapy and systemic approaches are the most common treatment options. Prognosis is dependent on location, subtype of lymphoma, and extent of systemic involvement.
Conclusion. The diagnosis, treatment and aftercare of ocular lymphoma requires a multidisciplinary approach between ophthalmology, hemato-oncology, radiotherapy, neurology, neurosurgery, oral and maxillofacial surgery, otorhinolaryngology, dermatology, radiology, pathology, and psycho-oncology. The ophthalmologist often acts as the primary contact person in this disease.

\section{Keywords}

Ocular adnexal lymphoma - Choroidal lymphoma - Iris lymphoma - Ciliary body lymphoma . Vitreoretinal lymphoma dung von lokalen Nebenwirkungen einer Strahlentherapie am Auge, wie einer starken und unangenehmen SiccaSymptomatik im Bindehautbereich oder einer Sehnervenschädigung im Optikusbereich, sowie eine vermutete geringere Rate an systemischen Rezidiven nach primär durchgeführter Chemotherapie [22]. Die Prognose der periokulären Lymphome hängt $a b$ von der Histologie, Tumorausbreitung und primär angewandten Therapie und ist in den meisten Fällen gut $[15,17,49]$.

\section{Uveale Lymphome}

Uveale Lymphome betreffen die Choroidea, den Ziliarkörper oder die Iris. Oft sind mehrere Strukturen betroffen. Z. B. dehnen sich Lymphome der Iris oder Choroidea nicht selten auf den Ziliarkörper aus. Den Hauptanteil der uvealen Lymphome macht der choroidale Befall aus [2, 11].

Beim choroidalen Lymphom sind Männer mit ca. $60 \%$ häufiger von der Erkrankung betroffen. Ein sekundärer Befall kommt in 5-31\% vor. Das pri- märe choroidale Lymphom stellt meist ein niedrigmalignes, unilaterales Lymphom von geringer Aggressivität dar. Klassische histologische Entität ist das EMZL [2, 32]. Initial suchen die Patienten den Augenarzt mit einer unklaren Sehverschlechterung auf. Dabei können ein Abfall des Visus, Verschwommenbzw. Nebelsehen auftreten. Der typische Befund lässt sich funduskopisch darstellen. Es zeigen sich weißlich gelbliche, zum Teil konfluierende Infiltrate der Aderhaut. Zudem muss der Ausschluss einer Uveitis posterior durch 


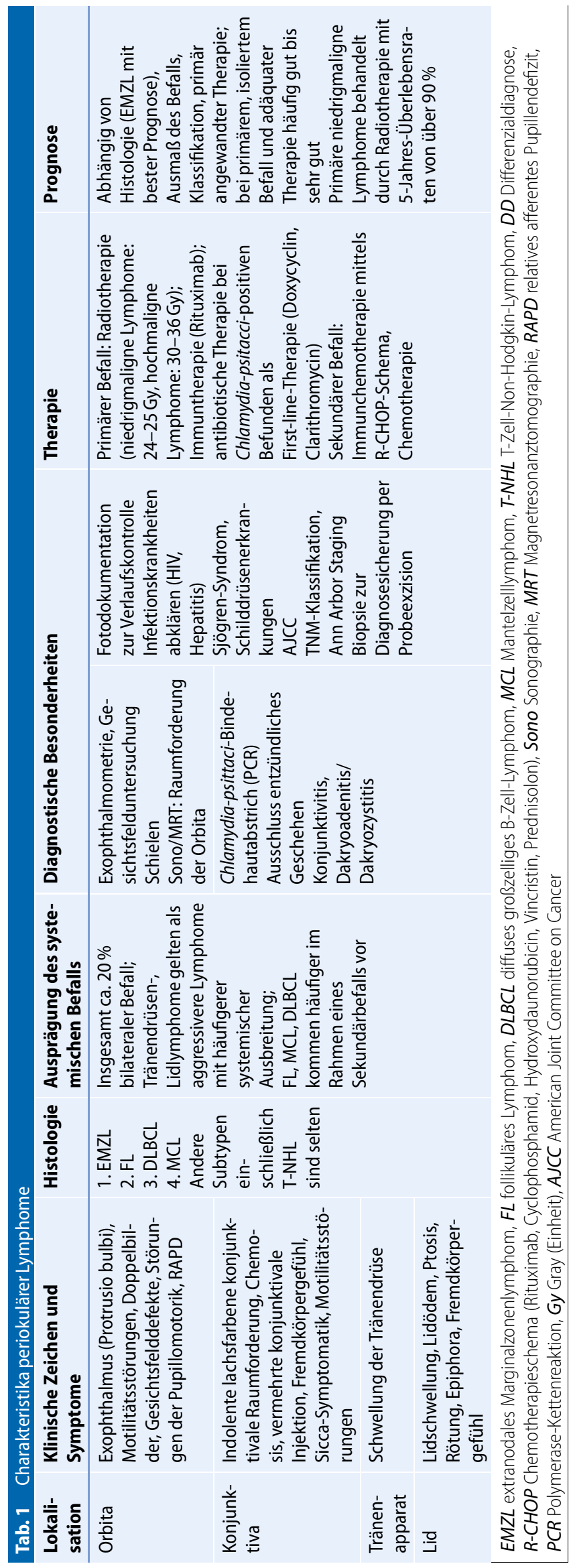

infektiologische bzw. rheumatische Laboruntersuchungen erfolgen [8, 32].

Beim Irislymphom dominiert ein sekundärer Befall in der Mehrzahl der Fälle. Pathohistologisch kommen hierbei gehäuft hochmaligne Formen wie das DLBCL oder das MCL vor [19]. Das Lymphom der Iris oder des Ziliarkörpers imponiert klinisch häufig als anteriore Uveitis bzw. Iritis. Typische klinische Zeichen sind neben der hauptbefundlichen weißlich flauschigen Raumforderung an der Iris ein Vorderkammerreizzustand mit Zellen sowie ein Tyndall-Phänomen als Zeichen einer Proteinausscheidung in das Kammerwasser. Am Ziliarkörper steht die Erhöhung des intraokulären Drucks im Vordergrund. Die Raumforderung kann mit einer Ausdehnung von bis $\mathrm{zu} 360^{\circ}$ auftreten $[8,31,46]$. Ein Ziliarkörpermelanom sollte differenzialdiagnostisch in Erwägung gezogen werden. Eine sichere Differenzierung ist nur durch eine Biopsie möglich.

Es sollte in jedem Fall eine Fotodokumentation der Befunde per Spaltlampen- bzw. Fundusfotografie erfolgen, um über die Tumorausbreitung das Ansprechen auf die Therapie beurteilen zu können. Bildgebende Untersuchungen wie Ultraschall und die optische Kohärenztomographie (OCT) können bei der Eingrenzung der Verdachtsdiagnose hilfreich sein. Zudem helfen beim choroidalen Lymphom die Fluoreszenzund Indozyaninangiographie bei der Abgrenzung vom White-dot-Syndrom $[2,11]$.

Um die Verdachtsdiagnose anschlieBend $\mathrm{zu}$ verifizieren, ist eine Biopsie erforderlich. Beim Iris- oder Ziliarkörperlymphom sollte dies als Probeexzision oder als Feinnadelaspiration erfolgen. Beim choroidalen Lymphom ist eine Aderhautbiopsie nötig. Neben der konventionellen histopathologischen und immunhistochemischen Untersuchung ist häufig eine molekularpathologische Analyse zum Klonalitätsnachweis für die Diagnosestellung erforderlich $[8,20$, 32].

Bei Primärmanifestation sollten sich hämatologische Untersuchungen wie u. a. eine Knochenmarkbiopsie anschließen. Beim uvealen Lymphom sollten in Rücksprache mit einem (Hämato-)On- 
Tab. 2 Charakteristika intraokulärer Lymphome

\begin{tabular}{|c|c|c|c|c|c|c|}
\hline $\begin{array}{l}\text { Lokali- } \\
\text { sation }\end{array}$ & $\begin{array}{l}\text { Klinische Zeichen } \\
\text { und Symptome }\end{array}$ & $\begin{array}{l}\text { Histo- } \\
\text { logie }\end{array}$ & $\begin{array}{l}\text { Ausprägung des } \\
\text { systemischen } \\
\text { Befalls }\end{array}$ & $\begin{array}{l}\text { Diagnostische Besonder- } \\
\text { heiten }\end{array}$ & Therapie & Prognose \\
\hline $\begin{array}{l}\text { Choro- } \\
\text { idea }\end{array}$ & $\begin{array}{l}\text { Visusabfall, Meta- } \\
\text { morphopsien, teils } \\
\text { auch Symptome } \\
\text { extraokulären Befalls }\end{array}$ & $\begin{array}{l}\text { Primär: } \\
\text { EMZL } \\
\text { Sekundär: } \\
\text { DLBCL }\end{array}$ & $\begin{array}{l}\text { Primärer Befall: } \\
\text { meist unilateral } \\
\text { Sekundärer Befall: } \\
\text { häufig bilateral } \\
\text { Primärer Befall } \\
\text { häufiger, sekun- } \\
\text { därer Befall in } \\
\text { 5-31\% }\end{array}$ & $\begin{array}{l}\text { Häufig auch extraokuläre } \\
\text { Strukturen (Konjunktiva) } \\
\text { befallen } \\
\text { Gelbliche, teils konfluieren- } \\
\text { de Infiltrate der Aderhaut } \\
\text { Infektiologisches/ } \\
\text { rheumatisches Labor } \\
\text { OCT, Angiographie } \\
\text { Aderhautbiopsie }\end{array}$ & \multirow[t]{3}{*}{$\begin{array}{l}\text { Chemotherapie, Rituximab, } \\
\text { Immunchemotherapie } \\
\text { (R-CHOP) } \\
\text { Ibrutinib } \\
\text { Radiotherapie (abhängig von } \\
\text { Malignität des Lymphoms } \\
\text { sowie eher bei primärem } \\
\text { Befall): } \\
\text { niedrigmaligne: } 28-36 \mathrm{~Gy} \\
\text { hochmaligne: } 30-40 \mathrm{~Gy}\end{array}$} & \multirow{3}{*}{$\begin{array}{l}\text { Stark abhängig } \\
\text { von Histologie } \\
\text { und Stadium, } \\
\text { primäres } \\
\text { choroidales } \\
\text { Lymphom mit } \\
\text { deutlich besserer } \\
\text { Prognose als } \\
\text { sekundärer Befall } \\
\text { Irislymphom } \\
\text { häufig mit } \\
\text { systemischem, } \\
\text { aggressivem } \\
\text { Verlauf }\end{array}$} \\
\hline Iris & $\begin{array}{l}\text { Raumforderung der } \\
\text { Iris, Vorderkammer- } \\
\text { reizzustand (Zel- } \\
\text { len+, Tyndall-Phä- } \\
\text { nomen + ), Erhöhung } \\
\text { des intraokulären } \\
\text { Drucks, Visusabfall, } \\
\text { Pseudohypopyon, } \\
\text { Hyphäma }\end{array}$ & $\begin{array}{l}\text { DLBCL, } \\
\text { MCL }\end{array}$ & $\begin{array}{l}\text { Normalerweise } \\
\text { unilateraler Befall } \\
\text { Sekundärer Befall } \\
\text { in der Mehrheit } \\
\text { der Fälle }\end{array}$ & $\begin{array}{l}\text { Vorderabschnitts-OCT } \\
\text { Blockexzision der Iris }\end{array}$ & & \\
\hline $\begin{array}{l}\text { Ziliar- } \\
\text { körper }\end{array}$ & $\begin{array}{l}\text { Erhöhung des in- } \\
\text { traokulären Drucks, } \\
\text { Schwellung des } \\
\text { Ziliarkörpers, Visus- } \\
\text { abfall, Iris bombata }\end{array}$ & $\begin{array}{l}\text { DLBCL, } \\
\text { MCL, } \\
\text { EMZL }\end{array}$ & $\begin{array}{l}\text { Isolierter Ziliar- } \\
\text { körperbefall zu } \\
\text { selten, dadurch } \\
\text { unzuverlässige } \\
\text { Datenlage }\end{array}$ & $\begin{array}{l}\text { Bis zu } 360^{\circ} \text {-ringförmige } \\
\text { oder partielle Raumforde- } \\
\text { rung des Ziliarkörpers }\end{array}$ & & \\
\hline $\begin{array}{l}\text { Retina/ } \\
\text { Glas- } \\
\text { körper }\end{array}$ & $\begin{array}{l}\text { Visusabfall, Mouches } \\
\text { volantes, Metamor- } \\
\text { phopsien, seröse } \\
\text { Netzhautablösung, } \\
\text { retinales Ödem, } \\
\text { retinale Gefäßver- } \\
\text { schlüsse }\end{array}$ & DLBCL & $\begin{array}{l}\text { Häufig primärer } \\
\text { Befall, PZNSL } \\
\text { initial in ca. } 20 \% \text {. } \\
\text { im Verlauf in } \\
\text { bis zu } 85 \% \text {; oft } \\
\text { initial oder im } \\
\text { Verlauf bilateral } \\
\text { auftretend }\end{array}$ & $\begin{array}{l}\text { Eventueller Befall des Vor- } \\
\text { derabschnitts } \\
\text { Glaskörper-Haze, „Aurora } \\
\text { borealis" } \\
\text { Gefäßeinscheidungen } \\
\text { orangene, weißlich bis } \\
\text { cremefarbene Herde retinal } \\
\text { oder subretinal } \\
\text { OCT, Autofluoreszenz, An- } \\
\text { giographie } \\
\text { Pars-plana-Vitrektomie }\end{array}$ & $\begin{array}{l}\text { Unilateraler Befall: intravi- } \\
\text { treale Therapie MTX oder } \\
\text { Rituximab oder Radiotherapie } \\
\text { (30-35 Gy) } \\
\text { Bilateral: intravitreale Therapie } \\
\text { und systemische Therapie } \\
\text { Vorliegen PZNSL: High-do- } \\
\text { se-Chemotherapie MTX } \\
\text { ggf. + intravitreale Therapie } \\
\text { lbrutinib }\end{array}$ & $\begin{array}{l}\text { Schlechteste } \\
\text { Prognose der } \\
\text { intraokulären } \\
\text { Lymphome } \\
\text { Mediane Überle- } \\
\text { benszeit } 44 \text { Mona- } \\
\text { te, gleichzeitiges } \\
\text { Vorliegen eines } \\
\text { PZNSL } 34 \text { Monate }\end{array}$ \\
\hline
\end{tabular}

kologen eine Schädel-MRT sowie eine Ganzkörper-CT erfolgen. Die MRT stellt den Goldstandard bei der Suche nach weiteren Manifestationen dar [11].

Es existiert aufgrund der geringen $\mathrm{Pa}$ tientenzahlen kein einheitliches Schema zur Behandlung uvealer Lymphome. Die systemische Therapie wird bei Irislymphomen und Ziliarkörperlymphomen meist in Erwägung gezogen aufgrund des häufigen systemischen Befalls [19]. Auch choroidale Lymphome mit ausgeprägtem Befallsmuster werden mit systemischer Therapie behandelt, da die Radiotherapie mit einer großflächigen Bestrahlung mit stärkeren Nebenwirkungen verbunden ist. Die systemische Gabe von Rituximab oder die Anwen- dung einer Immunchemotherapie ist möglich [2, 11].

Die Strahlentherapie kann vor allem bei primärem Befall erwogen werden, wird jedoch auch in Kombination mit der Chemotherapie bei systemischen Manifestationen angewandt [2, 31, 32]. Auch sekundäre Lymphome, welche die Sehkraft bedrohen, können in Kombination mit Immunchemotherapie bestrahlt werden [19]. Das Ausmaß des choroidalen Lymphoms spielt bei der Bestrahlung eine große Rolle in der Prognose des Visus des Patienten. Werden nur kleine Teile bestrahlt, kann die Prognose gut sein. Werden jedoch große Teile der Netzhaut bestrahlt, kann die Strahlentherapie durch eine Strahlenretinopathie oder -optikopathie auch zur Erblindung führen $[5,10]$. Die Chirurgie des Lymphoms ist auch hier meist nur diagnostischen Zwecken vorbehalten. Jedoch bleibt in manchen seltenen Fällen nur noch die Möglichkeit der Enukleation des Auges, um dem Patienten massive Schmerzen zu ersparen [2, 11, 31, 32].

$\mathrm{Da}$ die Prognose abhängig ist vom Stadium der Erkrankung und die Erkrankung eine Rarität darstellt, kommt einer personalisierten, tumorabhängigen Therapie eine große Bedeutung zu. Das Irislymphom, welches häufig in der Literatur als sekundäres, aggressives Lymphom beschrieben wird, hat eine schlechtere Prognose [18, 19, 31]. 


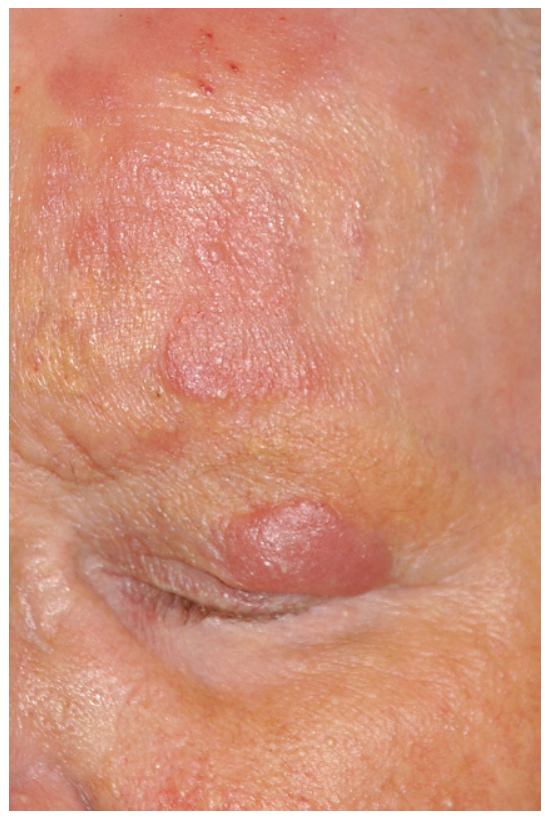

Abb. 2 ム Typisches Beispiel eines periokulären Lidlymphoms. Man sieht am linken Oberlid eine scharf begrenzte rötliche Plaque. Weitere Plaques sind auch an der Stirnregion sichtbar. Die Ursache ist ein primär kutanes T-Zell-Lymphom (CTCL) - das häufigste primär kutane Lymphom -, welches sich nunmehr im Plaquestadium befindet

\section{Vitreoretinale Lymphome}

Das primäre vitreoretinale Lymphom (PVRL) befällt die Strukturen der Netzhaut und des Glaskörpers. Es ist das häufigste primäre intraokuläre Lymphom und in den meisten Fällen als DLBCL zu klassifizieren [8, 16, 37]. Ein sekundärer (vitreo-)retinaler Befall ist selten. Das PVRL wird als besondere klinische Variante der primären Lymphome des zentralen Nervensystems (PZNSL) gesehen. Das PZNSL und das PVRL werden aufgrund ihrer biologischen und klinischen Besonderheiten von den systemischen DLBCL abgesetzt; eine systemische Dissemination außerhalb des ZNS ist selten [13]. Auge und ZNS werden gemeinsam mit dem Hoden als immunprivilegierte Lokalisationen betrachtet, in denen normale Mechanismen der Antigenerkennung und Immunantwort inaktiv sind [26, 40]. Interessanterweise zeigt das DLBCL des Hodens ein erhöhtes Risiko für eine Beteiligung bzw. Rezidive im ZNS und Auge [26]. Für PVRL wird eine Inzidenz von $0,017-0,048 / 100.000$ mit steigender
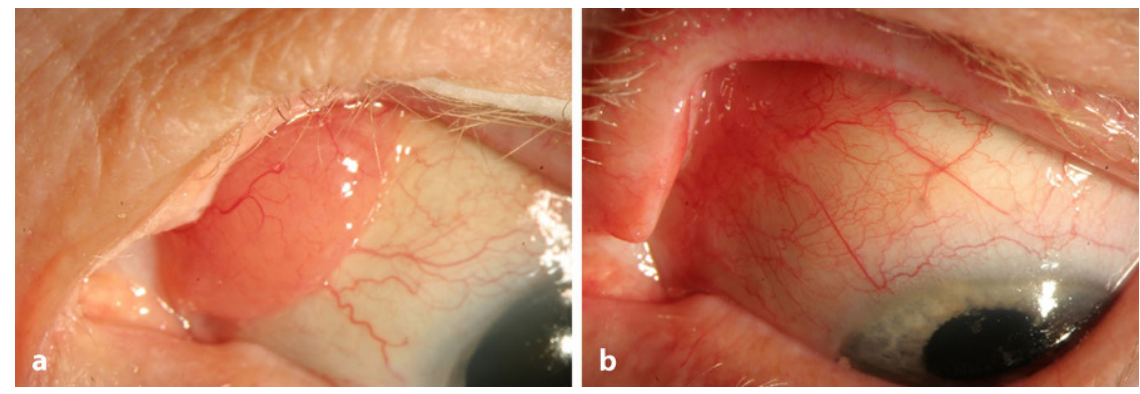

Abb. $3 \Delta$ Konjunktivales Lymphom vor und nach Bestrahlung. Dargestellt ist ein konjunktivales Lymphom des linken Auges (a), welches sich am inneren Lidwinkel manifestiert hat und als typische lachsfarbene Raumforderung in Erscheinung getreten ist. Der Tumor zeigt sich hier vaskularisiert und scharf abgrenzbar vom umliegenden Gewebe. Die histopathologische Untersuchung bestätigte ein MALT-Lymphom. Das systematische Staging ergab keinen weiteren Lymphombefall im Körper. b Status nach erfolgter Therapie. Das Lymphom ist auch fast ein Jahr nach Bestrahlung vollständig regredient. Verbliebene Anteile lassen sich nicht mehr vom umliegenden Gewebe abgrenzen. Nebenbefundlich zeigt sich in beiden Bildern eine Keratoconjunctivitis sicca

Tendenz angegeben. Bei PVRL besteht in bis zu $37 \%$ der Fälle bei Diagnosestellung gleichzeitig ein Befall des zentralen Nervensystems bzw. dieser tritt im Laufe der Erkrankung bei bis zu $85 \%$ der Patienten auf, umgekehrt entwickeln 15-25\% der Patienten mit PZNSL ein PVRL. Initial tritt das PVRL in bis zu $58 \%$ bilateral auf, im Verlauf der Erkrankung in bis zu $90 \%$ [30, 42]. Außer einer Infektion mit HIV sind keine Risikofaktoren für das PVRL bekannt $[8,12,16]$.

Erste Symptome des PVRL sind verschwommenes Sehen, abnehmende Sehschärfe oder Mouches volantes. Vom Auftreten der ersten Symptome bis zur Stellung der richtigen Diagnose vergehen im Durchschnitt bis zu 10 Monate. Bei gleichzeitigem Vorliegen eines PZNSL überwiegen oft die neurologischen Symptome. Typisch für das PVRL sind massive Trübungen im Glaskörper. Dies lässt zunächst an eine Vitritis im Rahmen einer posterioren Uveitis denken. Zu Beginn der Erkrankung können die Netzhautbefunde dezent sein und einer Vaskulitis ähneln, so wie in $\bullet$ Abb. 4 gezeigt. Im Verlauf treten orangene bis cremefarbene Lymphomansammlungen in der Netzhaut auf. Die weiteren klinischen Zeichen sind sehr vielfältig $[7,16,30]$.

Bei Verdacht auf ein PVRL sind anamnestisch neben der genauen Erhebung von Sehstörungen sowie der Dauer der Beschwerden vor allem Immunschwächen und vorhergehende hämatologische Erkrankungen zu evaluieren [7]. Anschließend soll eine ophthalmologi- sche Untersuchung erfolgen. Ebenfalls soll auf die o. g. Befunde geachtet werden. Auffälligkeiten sollen verlaufsdokumentiert werden. Als bildgebende Methoden werden die optische Kohärenztomographie und die Fluoreszenzangiographie eingesetzt. In der Sonographie am Auge können beispielsweise Glaskörperablagerungen, Veränderungen von Uvea und Sklera oder Netzhautablösungen objektiviert werden. Dies sind jedoch weniger spezifische Befunde für ein PVRL [16, 37, 45].

Es sollte eine Bildgebung des Kopfes stattfinden, um ein PZNSL zu detektieren. Die MRT stellt die Methode der Wahl dar [42]. Die endgültige Diagnosestellung erfolgt über eine Biopsie und eine anschließende pathomorphologische Aufarbeitung des Materials. Die Methode der Wahl stellt die Pars-plana-Vitrektomie dar, bei welcher durch kleinste Schnitte der Glaskörper entfernt wird [37]. Eine rasche Aufarbeitung des Materials bzw. eine Triage für die verschiedenen Untersuchungen einschließlich Zytologie, Immunzytochemie, Durchflusszytometrie, molekularer Analysen und Bestimmung von Zytokinspiegeln ist essenziell für eine erfolgreiche Diagnostik. Bei zellreichen Punktaten kann die Zellblocktechnik eingesetzt werden. Zytologisch zeigen sich große, atypische lymphatische Zellen, oft dominiert allerdings entweder eine hohe Anzahl von reaktiven, kleinen T-Zellen oder fortgeschrittene zytolytische Veränderungen mit schlechtem Erhaltungszustand. De- 


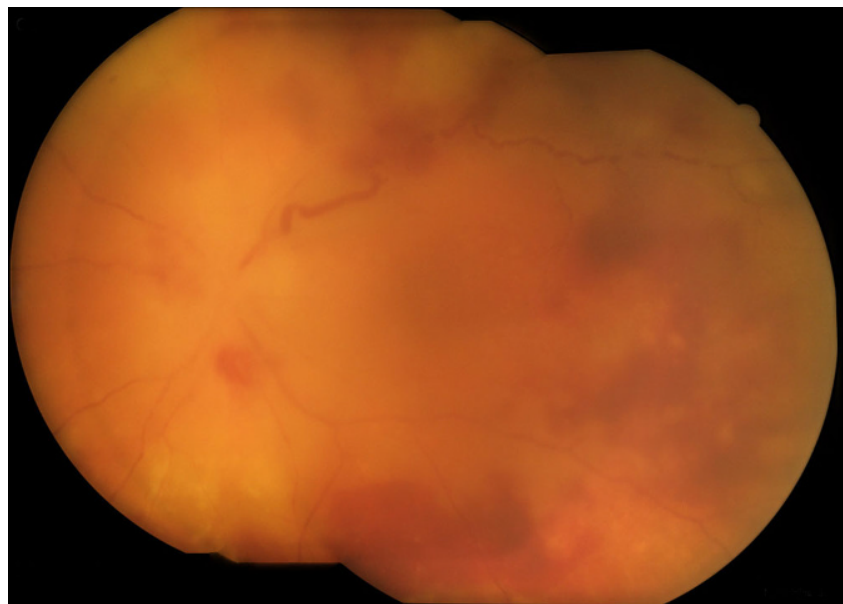

Abb. 4 ム Fundusfoto eines vitreoretinalen Lymphoms. Der funduskopische Einblick ist deutlich eingetrübt durch Infiltration des Glaskörpers (sog. „haze“) sowie der Netzhaut durch Lymphomzellen. Orange bis cremefarbene Ablagerungen können durch die Eintrübungen nur erahnt werden. Die Vene des oberen Gefäßbogens erscheint im Gegensatz zu den anderen Gefäßen verdickt. Zudem ist eine deutliche vaskulitisähnliche Gefäßzeichnung zu erkennen. Im Verlauf konnte ein PVRL histologisch mit einer Glaskörperprobe gesichert werden

generative Veränderungen, ein geringer Zellgehalt oder eine vorangegangene Steroidtherapie können die Diagnose sehr erschweren. Die Sensitivität der Zytologie wird mit $45-60 \%$ angegeben. Der immunzytochemische Nachweis von großen atypischen B-Zellen ist diagnostisch sehr wertvoll. In Fällen, bei denen die Vitrektomie keine eindeutige Diagnose erlaubt, kann eine chorioretinale Biopsie erfolgen. Molekulare Analysen zum Nachweis klonaler Immunglobulinumlagerungen mittels IgHPCR und IgL-PCR sind hilfreich, können aber aufgrund des immunprivilegierten Status des Auges mit wenigen B-Zellen einerseits und aufgrund der beim DLBCL oft ausgeprägten somatischen Hypermutation der rearrangierten IgGene andererseits sowohl falsch-positive als auch falsch-negative Ergebnisse liefern [12, 16, 20, 34]. Die Bestimmung von Zytokinspiegeln, speziell der "ratio“ zwischen IL-10 und IL-6, weist eine Sensitivität von 80 bis $90 \%$ und eine hohe Spezifität in der Abgrenzung zur Uveitis auf [48]. Trotz des breiten diagnostischen Methodenspektrums wird der falsch-negative Anteil der Vitrektomie beim PVRL auf ca. $30 \%$ geschätzt [7]. Eine Verbesserung der diagnostischen Trefferquote beim PVRL ist von zwei neuen molekularen Untersuchungen zu erwarten; speziell dem Nachweis re- kurrenter Mutationen und Alterationen im microRNA(miRNA)-Profil im Glaskörperaspirat. In einer Untersuchung konnten Mutationen von MYD88, das in $60-80 \%$ der Fälle von PZNSL alteriert ist, in $69 \%$ der Glaskörperproben vitreoretinaler Lymphome nachgewiesen und damit eine Steigerung der Sensitivität von $62 \%$ auf $90 \%$ erreicht werden [6]. Einige Studien beschäftigten sich mit Bestimmungen von microRNA aus Liquor oder Glaskörperbiopsaten als Biomarker zur Diagnostik von PZNSL und PVRL $[3,4,21]$. Insbesondere miRNA-91, -19b und -21 sind dabei vielversprechende Kandidaten [21].

Über die Therapie des PVRL wird in Abhängigkeit des Ausmaßes der Erkrankung entschieden. Liegt ein lokaler Befall vor, kommt es weiterhin darauf an, ob das Lymphom unilateral oder bilateral aufgetreten ist. Es stehen lokale Verfahren, wie die intravitreale Medikamentengabe von Methotrexat und/ oder Rituximab, zur Verfügung $[7,16]$. Die intravitreale Therapie hat sich durch ein günstiges Nebenwirkungsprofil etabliert [28]. Diese Medikamente können ebenfalls im Rahmen einer systemischen Therapie verabreicht werden [37]. Die Radiotherapie ist in der Lage, gute Ergebnisse in Bezug auf die Regression zu liefern, jedoch sind die Nebenwirkungen für das Augenlicht bedrohlicher [5,
7, 10]. Kombinierte Therapieformen aus systemischer Therapie und intravitrealer oder Strahlentherapie zeigten sich ebenfalls effektiv [9, 10, 27]. Im Jahr 2011 veröffentlichte die Primary Central Nervous System Lymphoma Collaborative Group Empfehlungen, welche vorsehen, für Patienten ohne systemischen Befall sowie ohne PZNSL bei unilateralem Befall eine intravitreale Therapie oder eine Strahlentherapie mit einer Dosis von 30 bis 35 Gy durchzuführen. Bei bilateralem Befall wird ebenfalls eine lokale Therapie empfohlen. Eine systemische Therapie bzw. eine Kombination einer intravitrealen Therapie mit einer systemischen Therapie sollte jedoch in Erwägung gezogen werden. Bei gleichzeitigem Vorliegen eines systemischen Befalls (PZNSL) wird eine Hochdosissystemtherapie u. U. mit intravitrealem Rituximab empfohlen. Eine Kombination mit einer intravitrealen Therapie kann aufgrund der schlechten okulären Penetration einer systemischen Therapie sinnvoll sein. Eine Bestrahlung des Auges sollte bei Versagen der anderen Therapie durchgeführt werden [7]. Unsere Empfehlungen für das Therapieregime weichen ein wenig von diesen doch älteren Empfehlungen ab und orientieren sich an neueren Studien $[9,27]$. Aufgrund neuster Daten und der allgemeinen internationalen Expertenmeinung wird sowohl beim isolierten PVRL als auch beim PVRL mit PZNSL eine Kombination aus systemischer und intravitrealer Therapie nach Maßgabe des Tumorboards empfohlen. Der Augenarzt ist gefordert, um das lokale Ansprechen der Therapie einzuschätzen und um frühzeitig auf ein mögliches Versagen reagieren zu können. Dabei stehen ihm die oben genannten diagnostischen Werkzeuge zur Verfügung. Speziell soll auf ophthalmologische Parameter geachtet werden. Unter Zuhilfenahme bildgebender Verfahren wie der MRT sollte auch regelmäßig der systemische Befall geprüft werden [7]. Zytokinlevelbestimmungen, insbesondere das Verhältnis von Interleukin-10 und -6, können auch im Follow-up wichtige Informationen liefern [20]. Die miRNA-Bestimmung und die Mutationsanalyse zellfreier DNA aus Glaskörperraumaspiraten könnten evtl. in der Zukunft auch bei 
der Nachsorge eine größere Rolle einnehmen [21].

Das PVRL zeigt von allen beschriebenen Lymphomen insgesamt die schlechteste Prognose. Gerade hier ist jedoch wieder das Ausmaß des Befalls entscheidend. Der gleichzeitige Befall mit einem PZNSL ist mit einer schlechteren Überlebensprognose verbunden [24, 39].

\section{Psychoonkologie/Nachsorge/ Leitlinien}

Die psychoonkologische Versorgung nimmt eine immer wichtigere Rolle in der modernen Medizin ein. Generell sollte bei jedem Patienten mit einem Lymphom, so auch Patienten mit okulärem Lymphom, der psychoonkologische Versorgungsbedarf ermittelt werden [29]. Die Psychoonkologie soll auch die Lebensqualität insgesamt steigern und leistet einen wichtigen Beitrag, den Patienten wieder in seinen Alltag zu integrieren. Starke Angst vor einem Rezidiv eines Lymphoms kann die Lebensqualität signifikant verschlechtern [25]. Verwiesen sei an dieser Stelle auf die S3-Leitlinie zur Psychoonkologie (https://www.awmf.org/leitlinien/detail/ 1l/032-051OL.html).

Die Nachsorgeuntersuchungen sollten der Schwere, Art und Lokalisation des Lymphoms angepasst werden und regelmäßig nach stattgehabter Therapie erfolgen. Nach Diagnose der Erkrankung und stattgehabter Therapie sollte 6 Wochen bis 3 Monate nach Therapieende eine Nachsorge erfolgen. Danach werden in den ersten beiden Jahren Zeiträume von drei Monaten empfohlen, welche dann bis zum fünften Jahr auf halbjährliche Kontrollen ausgedehnt werden können. Wichtig ist, wenn möglich die Fotodokumentation der Befunde, bildgebende Verfahren, grundlegende ophthalmologische Untersuchungen, die der Verlaufskontrolle dienen, angepasst an den anatomischen Befallsort sowie Laboruntersuchungen, um den Verlauf beurteilen zu können, in die Nachsorgeuntersuchungen einzubinden $[20,22]$.

Wichtig für eine adäquate Behandlung und Nachsorge ist ein leitliniengerechtes Vorgehen. Dabei ist hier auf die Leitlinien der Arbeitsgemeinschaft der Wis- senschaftlichen Medizinischen Fachgesellschaften e. V. (AWMF) (https://www. awmf.org/awmf-online-das-portal-derwissenschaftlichen-medizin/awmf-aktu ell.html) sowie der Deutschen Gesellschaft für Hämatologie und Medizinische Onkologie e.V. zu verweisen, welche auf https://www.onkopedia.com/ de/onkopedia/guidelines veröffentlicht werden.

\section{Fazit und Ausblick}

Klinische, biologische und therapeutische Faktoren verlangen beim okulären Lymphom eine multidisziplinäre Zusammenarbeit, in welcher der tumorerfahrene Augenarzt eine Schlüsselrolle einnimmt und häufig die erste Anlaufstelle für den Patienten ist. Er hat die diagnostischen Möglichkeiten, um eine Verdachtsdiagnose zu stellen, nicht selten, bevor in der Bildgebung ein Tumor zu erkennen ist. Die Erhaltung oder Verbesserung der Funktion des Auges ist nach der primären Tumorkontrolle ein maßgebliches und langfristiges Therapieziel. Nach Stellung der Verdachtsdiagnose und Biopsie durch den Augenarzt und histopathologischer Bestätigung eines Lymphoms sollte die weitere Vorstellung des Patienten in einer interdisziplinären Tumorkonferenz in die Wege geleitet werden, um eine personalisierte Therapiestrategie für jeden Patienten zu entwickeln. Die Therapie könnte bei allen Typen in Zukunft durch die sogenannten ,targeted therapies“ und „checkpoint inhibitors“ verbessert werden, bei denen es vor allem beim PVRL schon Ansätze gibt [18]. Erste Ergebnisse gibt es auch zur CART-Zell-Therapie bei Patienten mit ZNSLymphom [1]. Zukünftig könnte dieser Ansatz auch beim refraktären oder rezidivierenden PVRL zum Einsatz kommen. Bei jedem Patienten mit okulärem Lymphom sollte die Unterbringung und Behandlung innerhalb einer Studie geprüft werden.

\section{Korrespondenzadresse}

PD Dr. med. Vinodh Kakkassery

Klinik für Augenheilkunde, Universitätsklinikum Schleswig-Holstein, Campus Lübeck

Ratzeburger Allee 160, 23538 Lübeck,

Deutschland

vinodh.kakkassery@gmail.com

Förderung. Die Arbeit wurde zum Teil unterstützt durch OnkoTher-H, EU, ESF/14-BM-A55-0001/18, die Damp Stiftung 2017-05, DFG EM 63/13-1 und die Dermatologische Gesellschaft MecklenburgVorpommern (S. E.)

Funding. Open Access funding enabled and organized by Projekt DEAL.

\section{Einhaltung ethischer Richtlinien}

Interessenkonflikt. M. Zschoche, M. Ranjbar, L.M. Heindl, F. Fend und I.A. Adamietz geben an, dass kein Interessenkonflikt besteht. V. Kakkassery hat Vortragshonorare von den Firmen Roche, Bayer, Allergan und Novartis sowie Beratungshonorare von den Firmen Roche und Novartis erhalten. S. Emmert hat Berater- und Vortragshonorare von MSD, BMS, Pierre Fabre, Sanofi, Amgen, Novartis, LEO Pharma, Roche und Genzyme Corporation erhalten. N. von Bubnofferhielt Berater- und Vortragshonorare von AstraZeneca, Amgen, Novartis und BMS und Forschungsmittel von Novartis. S. Grisanti hat Vortragshonorare von Alcon, Allergan, Bayer, HumanOptics, Novartis, Pfizer und Roche erhalten, zudem hat er Beratungshonorare von Allergan, Bayer, Novartis, Pfizer, Roche und Zeiss erhalten. Er hat außerdem Forschungsmittel von Bayer, HumanOptics, Novartis, Pfizer, Roche, Santen, Second Sight, Thrombogenics, Transcende und Zeiss erhalten.

Für diesen Beitrag wurden von den Autoren keine Studien an Menschen oder Tieren durchgeführt. Für die aufgeführten Studien gelten die jeweils dort angegebenen ethischen Richtlinien.

Open Access. Dieser Artikel wird unter der Creative Commons Namensnennung 4.0 International Lizenz veröffentlicht, welche die Nutzung, Vervielfältigung, Bearbeitung, Verbreitung und Wiedergabe in jeglichem Medium und Format erlaubt, sofern Sie den/die ursprünglichen Autor(en) und die Quelle ordnungsgemäß nennen, einen Link zur Creative Commons Lizenz beifügen und angeben, ob Änderungen vorgenommen wurden.

Die in diesem Artikel enthaltenen Bilder und sonstiges Drittmaterial unterliegen ebenfalls der genannten Creative Commons Lizenz, sofern sich aus der Abbildungslegende nichts anderes ergibt. Sofern das betreffende Material nicht unter der genannten Creative Commons Lizenz steht und die betreffende Handlung nicht nach gesetzlichen Vorschriften erlaubt ist, ist für die oben aufgeführten Weiterverwendungen des Materials die Einwilligung des jeweiligen Rechteinhabers einzuholen.

Weitere Details zur Lizenz entnehmen Sie bitte der Lizenzinformation auf http://creativecommons.org/ licenses/by/4.0/deed.de. 


\section{Literatur}

1. Abramson JS, McGree B, Noyes $S$ et al (2017) Anti-CD19 CAR T cells in CNS diffuse large-B-cell lymphoma. N Engl J Med 377:783-784

2. Aronow ME, PortellCA, Sweetenham JWetal (2014) Uveal lymphoma: clinical features, diagnostic studies, treatment selection, and outcomes. Ophthalmology 121:334-341

3. Baraniskin A, Kuhnhenn J, Schlegel U et al (2011) Identification of microRNAs in the cerebrospinal fluid as biomarker for the diagnosis of glioma. Neuro-Oncology 14:29-33

4. Baraniskin A, Kuhnhenn J, Schlegel U et al (2012) MicroRNAs in cerebrospinal fluid as biomarker for disease course monitoring in primary central nervous system lymphoma. JNeurooncol 109:239-244

5. Berenbom A, Davila RM, Lin HS et al (2007) Treatment outcomes for primary intraocular lymphoma: implications for external beam radiotherapy. Eye 21:1198-1201

6. Bonzheim I, Giese S, Deuter C et al (2015) High frequency of MYD88 mutations in vitreoretinal B-cell lymphoma: a valuable tool to improve diagnostic yield of vitreous aspirates. Blood 126:76-79

7. Chan C-C, Rubenstein JL, Coupland SE et al (2011) Primary vitreoretinal lymphoma: a report from an International Primary Central Nervous System Lymphoma Collaborative Group symposium. Oncologist 16:1589-1599

8. Coupland SE, Damato B (2008) Understanding intraocular lymphomas. Clin Exp Ophthalmol 36:564-578

9. Damato B, Bever GJ, Kim DJ et al (2020) An audit of retinal lymphoma treatment at the University of California San Francisco. Eye 34:515-522

10. De La Fuente MI, Alderuccio JP, Reis IM et al (2019) Bilateral radiation therapy followed by methotrexate-based chemotherapy for primary vitreoretinallymphoma. Am JHematol 94:455-460

11. Doycheva D, Zierhut M, Süsskind D et al (2015) Diagnostik und Therapie der choroidalen Lymphome. Ophthalmologe 112:217-222

12. Fend F, Ferreri AJM, Coupland SE (2016) How we diagnose and treat vitreoretinal lymphoma. $\mathrm{Br} J$ Haematol 173:680-692

13. Ferreri AJM, Reni M, Zoldan MC et al (1996) Importance of complete staging in non-Hodgkin's lymphoma presenting as a cerebral mass lesion. Cancer 77:827-833

14. Goda JS, Gospodarowicz M, Pintilie $M$ et al (2010) Long-term outcome in localized extranodal mucosa-associated lymphoid tissue lymphomas treated with radiotherapy. Cancer 116:3815-3824

15. Graue GF, Finger PT, Maher E et al (2013) Ocular adnexal lymphoma staging and treatment: American Joint Committee on Cancer versus Ann Arbor. Eur J Ophthalmol 23:344-355

16. Jaehne D, CouplandSE (2018) Primäre vitreoretinale Lymphome. Ophthalmologe 115:343-356

17. Jenkins C, Rose GE, Bunce C et al (2003) Clinical features associated with survival of patients with lymphoma of the ocular adnexa. Eye (Lond) 17:809-820

18. Kakkassery V (2020) Personalisierte Medizin in der Behandlung von Augentumoren. Ophthalmologe 117:497-498

19. Kakkassery V, Coupland SE, Heindl LM (2020) Iris lymphoma-a systematic guide for diagnosis and treatment. Surv Ophthalmol. https://doi.org/10. 1016/j.survophthal.2020.06.003
20. Kakkassery V, Junemann AM, Bechrakis NE et al (2019) Ocular lymphoma: precise diagnostics and classification as key for successful personalized treatment. Ophthalmologe 117(6):499-507

21. Kakkassery V, Schroers R, Coupland SE et al (2017) Vitreous microRNA levels as diagnostic biomarkers for vitreoretinal lymphoma. Blood 129:3130-3133

22. Kakkassery V, Stubiger N, Adamietz IA et al (2015) Lymphoma of the ocular adnexa. Ophthalmologe 112:210-216

23. Kiesewetter B, Raderer M (2013) Antibiotic therapy in nongastrointestinal MALT lymphoma: a review of the literature. Blood 122:1350-1357

24. Kim MM, Dabaja BS, Medeiros J et al (2016) Survival outcomes of primary intraocular lymphoma: a single-institution experience. Am J Clin Oncol 39(2):109-113

25. Kim SJ, Kang D, Kim IR et al (2020) Impact of fear of cancer recurrence on survival among lymphoma patients. Psychooncology 29:364-372

26. King RL, Goodlad JR, Calaminici M et al (2020) Lymphomas arising in immune-privileged sites: insights into biology, diagnosis, and pathogenesis. Virchows Arch 476:647-665

27. Klimova A, Heissigerova J, Rihova E et al (2018) Combined treatment of primary vitreoretinal lymphomas significantly prolongs the time to first relapse. Br JOphthalmol 102:1579

28. Kvopka M, Lake SR, Smith JR (2020) Intraocular chemotherapy for vitreoretinal lymphoma: a review. Clin Exp Ophthalmol 48:240-248

29. Labouvie H, Hellmich S, Fath B et al (2019) Psychoonkologischer Versorgungsbedarf bei Patienten mit Non-Hodgkin-Lymphom. Onkologe 25:914-918

30. Levasseur SD, Wittenberg LA, White VA (2013) Vitreoretinal lymphoma: a 20-year review of incidence, clinical and cytologic features, treatment, and outcomes. JAMA Ophthalmol 131:50-55

31. Mashayekhi A, Shields CL, Shields JA (2013) Iris involvement by lymphoma: a review of 13 cases. Clin Exp Ophthalmol 41:19-26

32. Mashayekhi A, Shukla SY, Shields JA et al (2014) Choroidal lymphoma: clinical features and association with systemic lymphoma. Ophthalmology 121:342-351

33. McKelvie PA, McNab A, Francis IC et al (2001) Ocular adnexal lymphoproliferative disease: a series of 73 cases. Clin Exp Ophthalmol 29:387-393

34. Missotten T, Tielemans D, Bromberg JE et al (2013) Multicolor flowcytometric immunophenotyping is a valuable tool for detection of intraocular lymphoma. Ophthalmology 120:991-996

35. Moslehi R, Devesa SS, Schairer Cetal (2006) Rapidly increasing incidence of ocular non-hodgkin lymphoma. J Natl Cancer Inst 98:936-939

36. Moslehi R, Schymura MJ, NayakSetal (2011) Ocular adnexal non-Hodgkin's lymphoma: a review of epidemiology and risk factors. Expert Rev Ophthalmol 6:181-193

37. Pulido JS, Johnston PB, Nowakowski GS et al (2018) The diagnosis and treatment of primary vitreoretinal lymphoma: a review. Int J Retin Vitr 4:18

38. Richards H, Ramsden C, Naidoo R et al (2017) Ocular adnexal lymphomas: a review. Expert Rev Ophthalmol 12:133-148

39. Riemens A, Bromberg J, Touitou V et al (2015) Treatment strategies in primary vitreoretinal lymphoma: a 17-center European collaborative study. JAMA Ophthalmol 133:191-197

40. Riemens A, Missotten T, Baarsma S et al (2014) Diffuse large B-cell lymphoma in immunoprivileged sites: association of vitreoretinal, testicular and central nervous system lymphoma. Acta Ophthalmol 92:e159-e160

41. Robert Koch-Institut (2016) Bericht zum Krebsgeschehen in Deutschland 2016. Zentrum für Krebsregisterdaten im Robert Koch-Institut, Berlin

42. Stübiger N, Kakkassery V, Gundlach E et al (2015) Diagnostik und Therapie des primären vitreoretinalen Lymphoms. Ophthalmologe 112:223-230

43. Svendsen FH, Rasmussen PK, Coupland SE et al (2017) Lymphoma of the eyelid — an international multicenter retrospective study. Am J Ophthalmol 177:58-68

44. Tang L-J, Gu C-L, Zhang P (2017) Intraocular lymphoma. Int JOphthalmol 10:1301-1307

45. Ursea R, Heinemann MH, Silverman RH et al (1997) Ophthalmic, ultrasonographic findings in primary central nervous system lymphoma with ocular involvement. Retina 17:118-123

46. Valenzuela J, Yeaney GA, Hsi ED et al (2020) Large B-cell lymphoma of the uvea: histopathologic variants and clinicopathologic correlation. Surv Ophthalmol 65(3):361-370

47. Verdijk RM (2015) An update of ocular adnexal lymphomas. Diagn Histopathol 21:26-33

48. Wang Y, Shen D, Wang VM et al (2011) Molecular biomarkers for the diagnosis of primary vitreoretinal lymphoma. Int J Mol Sci 12:5684-5697

49. Zschoche M, Zimpfer A, Scheef BO et al (2020) Histopathological features and Ann Arbor stage in periocular lymphoma. In Vivo 34:1965-1974 